\title{
Fault Tolerant Formations Control of UAVs Subject to Permanent and Intermittent Faults
}

\author{
Qing Xu • Hao Yang • Bin Jiang • \\ Donghua Zhou • Youmin Zhang
}

Received: 31 August 2013 / Accepted: 13 September 2013 / Published online: 10 October 2013

(C) The Author(s) 2013. This article is published with open access at Springerlink.com

\begin{abstract}
The paper addresses the formation control of unmanned aerial vehicles (UAVs) in the presence of permanent and intermittent faults in each UAV. A fault tolerant control (FTC) scheme is developed to accommodate the permanent fault. It further shows that for the intermit-
\end{abstract}

This work is supported by National Natural Science Foundation of China (61034005, 61104116, 61273171, 61210012), and Doctoral Found of Ministry of Education of China (20113218110011).

Q. Xu $(\varangle) \cdot$ H. Yang · B. Jiang

College of Automation Engineering, Nanjing

University of Aeronautics and Astronautics,

Nanjing 210016, China

e-mail: 1521239691@qq.com

H. Yang

e-mail: haoyang@nuaa.edu.cn

B. Jiang

e-mail: binjiang@nuaa.edu.cn

D. Zhou

Department of Automation, Tsinghua University,

Beijing 100084, China

e-mail: ZDH@mail.tsinghua.edu.cn

\section{Y. Zhang}

Department of Mechanical and Industrial

Engineering, Concordia Institute of Aerospace Design

and Innovation, Concordia University,

1455 de Maisonneuve Blvd._W., Montreal,

Quebec H3G 1M8, Canada

e-mail: youmin.zhang@concordia.ca tent fault, the formation stability can be maintained under some conditions of fault appearance and disappearance without requiring to take any FTC action. Simulation results show the efficiency of the proposed method.

Keywords UAVs formation • Permanent fault • Intermittent fault $\cdot$ Fault tolerant control

\section{Introduction}

Formation flight of UAVs has attracted a great deal of interest in recent years. The structure of formation can be generally classified as leaderfollower, virtual-leader and so on [1]. At present, studies of UAVs formation commonly use simple first-order or second-order kinematic UAV model, which can not describe dynamic behaviors of UAVs in details. Moreover, these models cannot reflect the influences of UAV's own controller on formation flight and the type of fault as well.

On the other hand, a fault is an unpermitted deviation of at least one characteristic property or parameter of the system from the standard condition. The impact of a fault can be a small reduction in efficiency, but could also lead to overall system failure. Thus, an FTC scheme could have been designed to accommodate the fault. Faults can be classified according to their time characteristics as permanent and intermittent. They can also be 
classified according to their location of occurrence in the system as actuator faults, sensor faults and component faults [3].

As for the permanent faults, once they occur, they will exist in the system all the time. It is necessary to design a fault tolerant controller to stabilize the faulty system [4-7]. However, unlike permanent faults, the intermittent fault we pay attention to is a kind of fault that may be active at one instant of time causing a malfunction of system or may be inactive at another instant allowing the system to operate correctly. The intermittent fault often exists in electronic equipments and may be caused by noise, wind, magnetic or any other disturbance in the environment. It is well known that FTC takes time and cost. It is often not admissible to apply FTC scheme every time, since intermittent fault may occur frequently.

In this paper we consider the FTC problem of a UAVs formation in leader-follower structure, where each UAV may have both permanent and intermittent faults. Inspired by the idea proposed in [2], we divide the UAVs formation into outerloop and inner-loop: the outer-loop controls the whole formation; the inner-loop controls UAV's own dynamics and kinematics behavior.

The main contributions of this paper are as follows:

1. As for the permanent fault, a compensation term is added to the nominal controller of the UAV to eliminate the influences caused by the fault such that formation stability is still maintained.

2. The fault tolerance under intermittent faults is analyzed by the switched system approach. It shows that under some conditions of fault appearance and disappearance, the formation stability can be maintained without requiring to take any FTC action.

The rest of this paper is arranged as follows. Section 2 provides some preliminaries. Section 3 discusses the design method of inner-loop and outer-loop. Sections 4 and 5 respectively focus on the FTC design for permanent faults and intermittent faults. Section 6 provides simulation results, followed by conclusions in Section 7 .

\section{Preliminaries}

\subsection{Outer-Loop Model}

Consider the flight formation consists of $q(q \geq 2)$ UAVs, the topology considered here is leaderfollower structure, and each UAV has only one reference UAV. UAVi's kinematic model is

$$
\left\{\begin{array}{l}
\dot{x}_{i}=v_{i} \cos \phi_{i} \\
\dot{y}_{i}=v_{i} \sin \phi_{i} \\
\dot{\phi}_{i}=w_{i}, \quad i=1,2, \ldots, q
\end{array}\right.
$$

where $x_{i}, y_{i}$ represent UAV $i$ 's position, $v_{i}$ is forward speed, $\phi_{i}$ is the angle between $\mathrm{x}$-axis and $v_{i}$, $w_{i}$ are angular velocities.

In standard leader-follower formation model, forward error $\tilde{f}_{i j} \triangleq f_{i j}-f_{i j}^{d}$, lateral error $\tilde{l}_{i j} \triangleq l_{i j}-$ $l_{i j}^{d}, f_{i j}\left(l_{i j}\right), f_{i j}^{d}\left(l_{i j}^{d}\right)$ indicate actual and desired distance, as shown in Fig. 1. The information of $\mathrm{UAV} j$ are all known. The error model can be described as:

$$
\begin{aligned}
\tilde{f}_{i j}= & \left(x_{i}-x_{j}\right) \cos \phi_{j}+\left(y_{i}-y_{j}\right) \sin \phi_{j} \\
& +d \cos \left(\phi_{i}-\phi_{j}\right)-f_{i j}^{d} \\
\tilde{f}_{i j}= & \left(x_{i}-x_{j}\right) \sin \phi_{j}-\left(y_{i}-y_{j}\right) \cos \phi_{j} \\
& -d \sin \left(\phi_{i}-\phi_{j}\right)-l_{i j}^{d}
\end{aligned}
$$

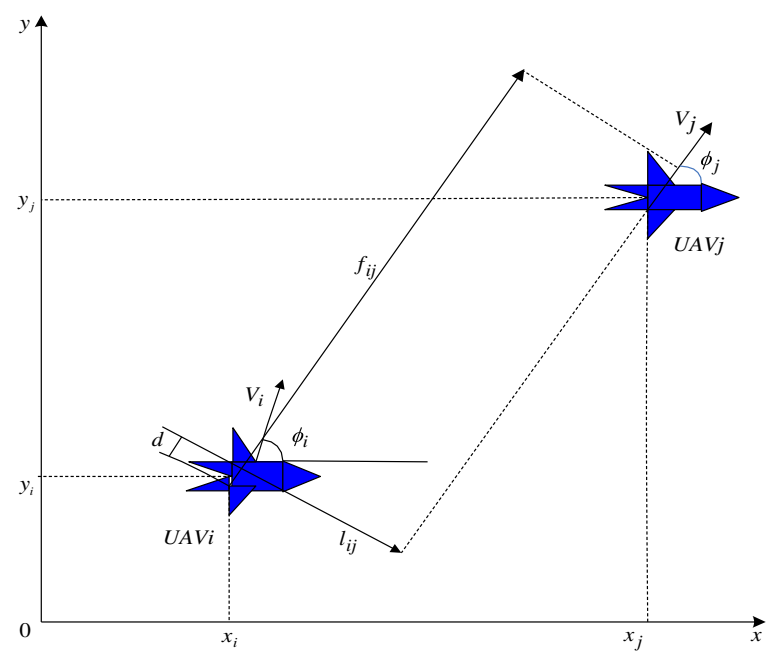

Fig. 1 Formation geometry 
Deriving Eq. 2 with regard to time leads to the dynamics of outer-loop model as follows:

$$
\begin{aligned}
{\left[\begin{array}{c}
\dot{\tilde{f}}_{i} \\
\tilde{\tilde{l}}_{i}
\end{array}\right]=} & {\left[\begin{array}{l}
-v_{j}-l_{i j} w_{j} \\
f_{i j} w_{j}
\end{array}\right] } \\
& +\left[\begin{array}{l}
\cos \left(\phi_{i}-\phi_{j}\right)-d \sin \left(\phi_{i}-\phi_{j}\right) \\
-\sin \left(\phi_{i}-\phi_{j}\right)-d \cos \left(\phi_{i}-\phi_{j}\right)
\end{array}\right]\left[\begin{array}{c}
v_{i} \\
w_{i}
\end{array}\right] \\
= & {\left[\begin{array}{l}
-v_{j}-l_{i j} w_{j} \\
f_{i j} w_{j}
\end{array}\right] } \\
& +\left[\begin{array}{l}
\cos \left(\phi_{i}-\phi_{j}\right)-d \sin \left(\phi_{i}-\phi_{j}\right) \\
-\sin \left(\phi_{i}-\phi_{j}\right)-d \cos \left(\phi_{i}-\phi_{j}\right)
\end{array}\right]\left[\begin{array}{c}
v_{i}^{*} \\
w_{i}^{*}
\end{array}\right] \\
& +\left[\begin{array}{l}
\cos \left(\phi_{i}-\phi_{j}\right)-d \sin \left(\phi_{i}-\phi_{j}\right) \\
-\sin \left(\phi_{i}-\phi_{j}\right)-d \cos \left(\phi_{i}-\phi_{j}\right)
\end{array}\right] \\
& \times\left[\begin{array}{l}
v_{i}-v_{i}^{*} \\
w_{i}-w_{i}^{*}
\end{array}\right]
\end{aligned}
$$

Note that $v_{i}$ and $w_{i}$ of UAV $i$ can be regarded as the inputs of outer-loop model, $v_{i}^{*}, w_{i}^{*}$ in Eq. 3 are the virtual and ideal control laws. Designing $v_{i}^{*}, w_{i}^{*}$ properly can make the system

$$
\begin{aligned}
{\left[\begin{array}{c}
\dot{\tilde{f}}_{i} \\
\dot{\tilde{l}}_{i}
\end{array}\right]=} & {\left[\begin{array}{l}
-v_{j}-l_{i j} w_{j} \\
f_{i j} w_{j}
\end{array}\right] } \\
& +\left[\begin{array}{l}
\cos \left(\phi_{i}-\phi_{j}\right)-d \sin \left(\phi_{i}-\phi_{j}\right) \\
-\sin \left(\phi_{i}-\phi_{j}\right)-d \cos \left(\phi_{i}-\phi_{j}\right)
\end{array}\right]\left[\begin{array}{c}
v_{i}^{*} \\
w_{i}^{*}
\end{array}\right]
\end{aligned}
$$

stable. This will be discussed in Section 3 .

\subsection{Inner-Loop Model}

Different from outer-loop model, the inner-loop model describes UAVs' own dynamical and kinematical behavior, and can be written as follows

$$
\left\{\begin{array}{l}
\dot{m}_{i}(t)=A m_{i}(t)+B \delta_{i}(t) \\
n_{i}(t)=C m_{i}(t)=\left[\begin{array}{ll}
v_{i} & \psi_{i}-\beta_{i}
\end{array}\right] \\
z_{i}(t)=D \dot{n}_{i}(t)=\left[\begin{array}{l}
\dot{\psi}_{i}-\dot{\beta}_{i}
\end{array}\right]
\end{array}\right.
$$

where $m_{i}=\left[\begin{array}{llllllll}v_{i} & \alpha_{i} & q_{i} & \theta_{i} & \beta_{i} & p_{i} & r_{i} & \psi_{i}\end{array}\right]^{T} \in \mathbb{R}^{8}$ are state variables. They are forward velocity, angle of attack, pitch rate, pitch angle, slideslip angle, roll rate, yaw rate and yaw angle. The input variables of inner-loop are $\delta_{i}=\left[\begin{array}{llll}\delta_{i e} & \delta_{i T} & \delta_{i a} & \delta_{i r}\end{array}\right]^{T} \in \mathbb{R}^{4}$, indicating elevator, throttle, flap and aileron. They are UAV's real physical control variables. $n_{i}(t), z_{i}(t)$ are both output variables, and $z_{i}(t)=\left[\dot{\psi}_{i}-\dot{\beta}_{i}\right]=w_{i}$. Note that the inputs $v_{i}, w_{i}$ of outer-loop are the same as the outputs $v_{i}, \dot{\psi}_{i}-\dot{\beta}_{i}$ of the inner-loop. $A, B$, $C$ are matrices of certain dimension. $m_{i}(0)$ is the initial state of the system.

\subsection{Models of Faults \\ 2.3.1 Permanent Fault Model}

The model of permanent fault discussed here is actuator fault. The type of fault under consideration is the loss of actuator effectiveness. Let $\delta_{i}^{F}(t)$ represent the signal from the $i$ th actuator that has failed. Then the permanent fault can be described as follows:

$\delta_{i}^{F}(t)=\rho_{i} \delta_{i}(t)$

$\rho_{i}=\operatorname{diag}\left[\rho_{i 1}, \quad \rho_{i 2}, \quad \rho_{i 3}, \rho_{i 4}\right]$

where $0<\rho_{i j} \leq 1, j=1,2,3,4$. If $\rho_{i}$ equals to a unit matrix, there is no fault.

\subsubsection{Intermittent Fault Model}

The intermittent fault consider here occurs in the output channels of the onboard control processor, under which the inner-loop model changes into

$\dot{m}_{i}(t)=A m_{i}(t)+B f_{i} \delta_{i}(t)$

where

$f_{i}= \begin{cases}f & \text { otherwise } \\ 1 & \text { the system is fault free }\end{cases}$

with $0<f<1$.

A realistic model to represent the appearance and disappearance property of intermittent fault is continuous-parameter Markov chain $[9,10]$ as shown in Fig. 2. Mode " 0 " and "1" represent healthy and faulty situations respectively. The probability for going from 0 to 1 at any time is $\lambda$, and the probability for going from 1 to 0 at any time is $\mu$. The equations for these probabilities are [15-17]

$$
\left\{\begin{array}{l}
P\left\{f_{i}(t+\Delta t)=f \mid f_{i}(t)=1\right\}=\lambda \Delta t \\
P\left\{f_{i}(t+\Delta t)=1 \mid f_{i}(t)=0\right\}=\mu \Delta t
\end{array}\right.
$$




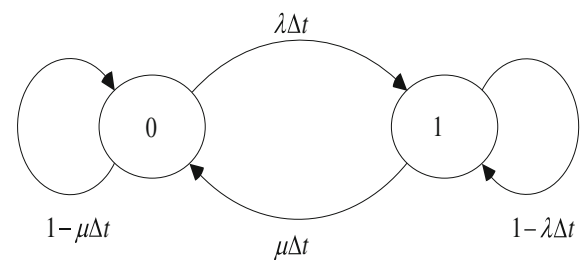

Fig. 2 Continuous two-state model

where $0 \leq \lambda<1$ represent the fault appearance rates, $0 \leq \mu<1$ represent the fault disappearance rates. $\Delta t \geq 0$ is the infinitesimal transition time interval. Assume that the initial situation is healthy.

\subsection{Problem Formulation}

According to the analysis in Section 2.2, one can obtain that $w_{i}=\dot{\psi}_{i}-\dot{\beta}_{i}$. If $\psi_{i}-\beta_{i}$ can track the given signal, then $\dot{\psi}_{i}-\dot{\beta}_{i}$ can track $w_{i}^{*}$ as well. The ultimate goal is to design $\delta_{i}(t)$ in Eq. 4 to as sure that the error system (3) is asymptotically stable in both normal and faulty situations.

Given a reference signal $y_{i r}(t)$, define

$\varepsilon_{i}(t) \triangleq y_{i r}(t)-S n_{i}(t)=\left[\begin{array}{l}v_{i}-v_{i}^{*} \\ \psi_{i}-\psi_{i}^{*}-\left(\beta_{i}-\beta_{i}^{*}\right)\end{array}\right]$

where $S \in \mathrm{R}^{2 \times 2}$ is a known constant matrix, $\psi_{i}^{*}, \beta_{i}^{*}$ are desired values.

Let $\eta_{i}(t)=\int_{0}^{t} \varepsilon_{i}(\tau) d \tau, \bar{m}_{i}(t)=\left[\begin{array}{ll}\eta_{i}^{T}(t) & m_{i}^{T}(t)\end{array}\right]^{T}$ (4) and (5) can be combined and the following augmented system can be obtained:

$$
\begin{aligned}
{\left[\begin{array}{c}
\dot{\eta}_{i}(t) \\
\dot{m}_{i}(t)
\end{array}\right]=} & {\left[\begin{array}{cc}
0 & -S C \\
0 & A
\end{array}\right]\left[\begin{array}{c}
\eta_{i}(t) \\
m_{i}(t)
\end{array}\right]+\left[\begin{array}{l}
0 \\
B
\end{array}\right] \delta_{i}(t) } \\
& +\left[\begin{array}{l}
I \\
0
\end{array}\right] y_{i r}(t)
\end{aligned}
$$

Where the augmented system can be described as:

$$
\begin{aligned}
& \left\{\begin{array}{l}
\dot{\bar{m}}_{i}(t)=\bar{A}_{\bar{m}_{i}}(t)+\bar{B} \delta_{i}(t)+G y_{i r}(t) \\
\bar{m}_{i}(0)=\bar{m}_{i 0}
\end{array}\right. \\
& \bar{A}=\left[\begin{array}{cc}
0 & -S C \\
0 & A
\end{array}\right], \bar{B}=\left[\begin{array}{l}
0 \\
B
\end{array}\right], G=\left[\begin{array}{c}
I_{2 \times 2} \\
0
\end{array}\right] .
\end{aligned}
$$

$\bar{m}_{i}(0)$ is the initial state. At the occurrence of a fault, the purpose of FTC scheme is to make $\lim _{t \rightarrow \infty} \varepsilon_{i}(t)=0$ and variables of $m_{i}(t)$ bounded.

This work assumes that the appearance and disappearance of the fault can be detected rapidly by using a certain fault diagnosis scheme which is not the main focus of the paper. Interested readers are referred to, e.g., [3, 8, 11, 12] for detailed information.

\section{Design of Controller}

First of all, we design $v_{i}^{*}, w_{i}^{*}$ for outer-loop model (3). Then the controller $\delta_{i}$ is designed for the inner-loop system to make sure that the state variables $m_{i}$ are bounded and $v_{i}, w_{i}$ can track $v_{i}^{*}, w_{i}^{*}$.

\subsection{Outer-Loop Controller Design}

For the designed communication topology, UAV $i$ has only one reference vehicle $j$. The desired $v_{i}^{*}, w_{i}^{*}$ are as follows

$\left[\begin{array}{c}v_{i}^{*} \\ w_{i}^{*}\end{array}\right]=\left[\begin{array}{cc}\cos \tilde{\phi}_{i} & -\sin \tilde{\phi}_{i} \\ -\frac{1}{d} \sin \tilde{\phi}_{i} & -\frac{1}{d} \cos \tilde{\phi}_{i}\end{array}\right]\left[\begin{array}{c}-k_{1} \tilde{f}_{i}+v_{j}+l_{i j} w_{j} \\ -k_{2} \tilde{l}_{i}-f_{i j} w_{j}\end{array}\right]$

Where $\quad \tilde{\phi}_{i} \triangleq \phi_{i}-\phi_{j}, \tilde{f}_{i} \triangleq f_{i j}-f_{i j}^{d}, \tilde{l}_{i} \triangleq l_{i j}-l_{i j}^{d}$, $k_{1}, k_{2}>0$ are feedback gain.

Apply Eqs. 10 to 3, one can get

$$
\begin{aligned}
{\left[\begin{array}{c}
\dot{\tilde{f}}_{i} \\
\dot{\tilde{l}}_{i}
\end{array}\right]=} & {\left[\begin{array}{c}
-k_{1} \tilde{f}_{i} \\
-k_{2} \tilde{l}_{i}
\end{array}\right]\left[\begin{array}{c}
\tilde{f}_{i} \\
\tilde{l}_{i}
\end{array}\right] } \\
& +\left[\begin{array}{cc}
\cos \tilde{\phi}_{i} & -d \sin \tilde{\phi}_{i} \\
-\sin \tilde{\phi}_{i} & -d \cos \tilde{\phi}_{i}
\end{array}\right]\left[\begin{array}{c}
v_{i}-v_{i}^{*} \\
w_{i}-w_{i}^{*}
\end{array}\right]
\end{aligned}
$$

Obviously, the above system is input-to-state stable with regard to $\left[\begin{array}{c}v_{i}-v_{i}^{*} \\ w_{i}-w_{i}^{*}\end{array}\right][19-22]$. If the inner-loop controller can ensure that $\left[\begin{array}{c}v_{i}-v_{i}^{*} \\ w_{i}-w_{i}^{*}\end{array}\right]$ approaches 0 , then the formation error converges to 0 . 
3.2 Inner-Loop Controller Design

In order to make the inner-loop outputs track the given ideal signal $\left[v_{i}^{*} w_{i}^{*}\right]^{T}$, we design the following feedback controller of nominal system (10):

$$
\delta_{i 1}(t)=K_{i 1} \bar{m}_{i}(t)=\left[\begin{array}{ll}
K_{i 1 \eta_{i}} & K_{i 1 m_{i}}
\end{array}\right]\left[\begin{array}{c}
\eta_{i}(t) \\
m_{i}(t)
\end{array}\right] \triangleq \delta_{i 1}(t)
$$

where $K_{i 1}$ is the feedback gain of the controller, then the closed-loop system can be described as:

$\dot{\bar{m}}_{i}(t)=\left(\bar{A}+\bar{B} K_{i 1}\right) \bar{m}_{i}(t)+G y_{i r}(t)$

Lemma 1 For a given constant $\gamma$, if there exist symmetric matrices $Z \in \mathbb{R}^{10 \times 10}$ and $W \in \mathbb{R}^{4 \times 10}$ such that the following linear matrix inequality (14) holds

$$
\left[\begin{array}{ccccc}
\bar{A} Z+Z \bar{A}^{T}+\bar{B} W+W^{T} \bar{B}^{T} & G & W^{T} R^{1 / 2} & Z Q^{1 / 2} & \\
* & -\gamma I & & 0 & 0 \\
* & * & -I & 0 & \\
* & * & * & -I
\end{array}\right]<0
$$

Then there exists control law $\delta_{i 1}(t)=K_{i 1} \bar{m}_{i}(t)$, $K_{i 1}=W Z^{-1}$, such that $\eta_{i}$ is asymptotically stable, and $m_{i}$ is bounded.

Proof The proof is similar to [14], and thus is omitted.

Lemma 2 (Bellman-Gronwall) If some numbers $t_{b} \geq t_{a}$, some constant $C \geq 0, N \geq 0$, and some non-negative, piecewise-continuous-function $g$ : $\left[t_{a}, t_{b}\right] \rightarrow \mathbb{R}, w:\left[t_{a}, t_{b}\right] \rightarrow \mathbb{R}$ is a continuous function satisfying

$|w(t)| \leq C+N \int_{t_{a}}^{t}|g(\tau)||w(\tau)| d \tau, \quad t \in\left[\begin{array}{ll}t_{a}, & t_{b}\end{array}\right]$

Then

$|w(t)| \leq C e^{N \int_{t a}^{t}|g(\tau)| d \tau} \quad t \in\left[t_{a}, t_{b}\right]$

Proof The proof is similar to [25], and thus is omitted.

Theorem 1 If there exists a symmetric matrix $P_{i}$ such that the following inequality

$A^{T} P_{i}+P_{i} A+\left(B K_{i 1}\right)^{T} P_{i}+P_{i} B K_{i 1}<0$

holds, then the error $\eta_{i}$ is exponential stable and $m_{i}$ is bounded.
Proof We choose a Lyapunov function for $\eta_{i}$ as $V_{i}=\eta_{i}^{T}(t) P_{i} \eta_{i}(t)$

The time derivative of $V_{i}$ along the solution of Eq. 10 with Eq. 13 is

$$
\begin{aligned}
\dot{V}_{i}(t)= & \dot{\eta}_{i}^{T}(t) P_{i} \eta_{i}(t)+\eta_{i}^{T}(t) P_{i} \dot{\eta}_{i}(t) \\
= & {\left[\left(A_{\eta_{i}}+B_{\eta_{i}} K_{i 1}\right) \eta_{i}(t)+G_{\eta_{i}} y_{r}(t)\right]^{T} P_{i} \eta_{i}(t) } \\
& +\eta_{i}^{T}(t) P_{i}\left[\left(A_{\eta_{i}}+B_{\eta_{i}} K_{i 1}\right) \eta_{i}(t)+G_{\eta_{i}} y_{i r}(t)\right] \\
= & \eta_{i}^{T}(t) A_{\eta_{i}}^{T} P_{i} \eta_{i}(t)+\eta_{i}(t)\left(B_{\eta_{i}} K_{i 1}\right)^{T} P_{i} \eta_{i}(t) \\
& +y_{i r}^{T} G_{\eta_{i}}^{T}(t) P_{i} \eta_{i}(t)+\eta_{i}^{T}(t) P_{i} A_{\eta_{i}} \eta_{i}(t) \\
& +\eta_{i}^{T}(t) P_{i} B_{\eta_{i}} K_{i 1} \eta_{i}(t)+\eta_{i}^{T} P_{i} G_{\eta_{i}} y_{i r}(t) \\
= & \eta_{i}^{T}(t)\left(A_{\eta_{i}} P_{i}+P_{i} A_{\eta_{i}}\right) \eta_{i}(t) \\
& +\eta_{i}^{T}(t)\left[\left(B_{\eta_{i}} K_{i 1}\right)^{T} P_{i}+P_{i} B_{\eta_{i}} K_{i 1}\right] \eta_{i}(t) \\
& +G_{\eta_{i}}^{T} y_{i r}^{T}(t) P_{i} \eta_{i}(t)+\eta_{i}^{T}(t) P_{i} G_{\eta_{i}} y_{i r}(t) \\
= & \eta_{i}^{T}(t)\left[A_{\eta_{i}}^{T} P_{i}+P_{i} A_{\eta_{i}}+\left(B_{\eta_{i}} K_{i 1}\right)^{T} P_{i}\right. \\
& \left.\quad+P_{i} B_{\eta_{i}} K_{i 1}\right] \eta_{i}(t)+2 \eta_{i}^{T}(t) P_{i} G_{\eta_{i}} y_{i r}(t) \\
\leq & \eta_{i}^{T}(t)\left[A_{\eta_{i}}^{T} P_{i}+P_{i} A_{\eta_{i}}\right. \\
& \left.\quad+\left(B_{\eta_{i}} K_{i 1}\right)^{T} P_{i}+P_{i} B_{\eta_{i}} K_{i 1}\right] \eta_{i}(t) \\
& +2\left|\eta_{i}^{T}(t)\right| P_{i} G_{\eta_{i}}\left|y_{i r}(t)\right| \\
\leq & \eta_{i}^{T}(t)\left[A_{\eta_{i}}^{T} P_{i}+P_{i} A_{\eta_{i}}+\left(B_{\eta_{i}} K_{i 1}\right)_{i}^{T} P\right. \\
& \left.\quad+P_{i} B_{\eta_{i}} K_{i 1}\right] \eta_{i}(t)
\end{aligned}
$$


It follows that

$A_{\eta_{i}}^{T} P_{i}+P_{i} A_{\eta_{i}}+\left(B_{\eta_{i}} K_{i 1}\right)^{T} P_{i}+P_{i} B_{\eta_{i}} K_{i 1}<0$

Let

$A_{\eta_{i}}^{T} P_{i}+P_{i} A_{\eta_{i}}+\left(B_{\eta_{i}} K_{i 1}\right)^{T} P_{i}+P_{i} B_{\eta_{i}} K_{i 1}=-Q_{i}$

We further have

$\dot{V}_{i} \leq-\lambda_{i 1}\left\|\eta_{i}(t)\right\|^{2}$

where $\lambda_{i 1}>0$ is the eigenvalue of $Q_{i}$, which means that the error $\eta_{i}(t)$ of $\bar{m}_{i}(t)$ is exponential stable.

As for $m_{i}(t)$ of $\bar{m}_{i}(t)$, the reference signal $y_{i r}(t)$ is not considered at first, then

$\dot{m}_{i}(t)=\bar{A}_{m_{i}} m_{i}(t)+\bar{B}_{m_{i}} \delta_{i}(t)$

Assuming that the station transition matrix of closed-loop system (17) is $\phi_{i}(t)=e^{\bar{A}_{m_{i}} t}$, and the following inequality holds

$\left\|\phi_{i}(t)\right\|=\left\|e^{\bar{A}_{m_{i}} t}\right\| \leq m_{0} e^{-\alpha t}, \alpha>0, \forall t \geq 0$

the solution of Eq. 17 can be described as:

$$
\begin{aligned}
m_{i}(t)= & \phi_{i}(t, 0) m_{i}(0)+\int_{0}^{t} \phi_{i}(t-\tau) \bar{B}_{m_{i}} \delta_{i}(\tau) d \tau \\
\leq & \left\|\phi_{i}(t)\right\|\left\|m_{i}(0)\right\| \\
& +\int_{0}^{t}\left\|\phi_{i}(t-\tau)\right\|\left\|\bar{B}_{m_{i}}\right\|\left\|\delta_{i}(\tau)\right\| d \tau \\
\leq & \left\|\phi_{i}(t)\right\|\left\|m_{i}(0)\right\| \\
& +\int_{0}^{t}\left\|\phi_{i}(t-\tau)\right\|\left\|\bar{B}_{m_{i}}\right\|\left\|K_{i 1_{m_{i}}} m_{i}(\tau)\right\| d \tau \\
\leq & \left\|\phi_{i}(t)\right\|\left\|m_{i}(0)\right\| \\
& +\int_{0}^{t}\left\|\phi_{i}(t-\tau)\right\|\left\|\bar{B}_{m_{i}} K_{i 1_{m_{i}}}\right\|\left\|m_{i}(\tau)\right\| d \tau
\end{aligned}
$$

$\leq\left\|\phi_{i}(t)\right\|\left\|m_{i}(0)\right\|$

$$
+\int_{0}^{t}\left\|\phi_{i}(t-\tau)\right\| \beta_{A}\left\|m_{i}(\tau)\right\| d \tau
$$

$\left\|m_{i}(t)\right\| \leq m_{0} e^{-\alpha t}\left\|m_{i}(0)\right\|$

$$
+\int_{0}^{t} m_{0} e^{-\alpha(t-\tau)} \beta_{A}\left\|m_{i}(\tau)\right\| d \tau
$$

$\left\|m_{i}(t)\right\| e^{\alpha t} \leq m_{0}\left\|m_{i}(0)\right\|$

$$
+\int_{0}^{t} m_{0} e^{\alpha \tau} \beta_{A}\left\|m_{i}(\tau)\right\| d \tau
$$

According to Bellman-Gronwall Lemma, we have

$$
\begin{aligned}
\left\|m_{i}(t)\right\| e^{\alpha t} & \leq m_{0}\left\|m_{i}(0)\right\| e^{m_{0} \beta_{A} \int_{0}^{t} d t} \\
& \leq m_{0}\left\|m_{i}(0)\right\| e^{m_{0} \beta_{A} t} \\
\left\|m_{i}(t)\right\| & \leq m_{0}\left\|m_{i}(0)\right\| e^{-\left(\alpha-m_{0} \beta_{A}\right) t}
\end{aligned}
$$

While $-\left(\alpha-m_{0} \beta_{A}\right)<0,\left\|m_{i}(t)\right\|$ is bounded. Then take the reference $y_{i r}(t)$ into consideration, $y_{i r}(t)$ and matrix $G$ are known, they are all bounded, so $\left\|m_{i}(t)\right\|$ is still bounded.

According to the above analysis, one can see that the error $\eta_{i}$ of the inner-loop system is exponential stable and the states $m_{i}$ of inner-loop system are bounded. The proof is completed.

\section{FTC of Permanent Faults}

\subsection{The Control Strategy}

Once a permanent fault occurs, a fault tolerant controller is needed to re-stabilize the system. Actuator fault is one of common permanent faults.

The augmented system (10) with permanent fault can be described as:

$$
\left\{\begin{array}{l}
\dot{\bar{m}}_{i}(t)=\bar{A} \bar{m}_{i}(t)+\bar{B} \rho_{i} \delta_{i}(t)+G y_{i r}(t) \\
n_{i}(t)=C \bar{m}_{i}(t) \\
z_{i}(t)=D \dot{n}_{i}(t)=\left[\dot{\psi}_{i}-\dot{\psi}_{i}^{*}-\left(\dot{\beta}_{i}-\dot{\beta}_{i}^{*}\right)\right]
\end{array}\right.
$$

If $\psi_{i}, \beta_{i}$ track the signal $y_{i r}(t), \dot{\psi}_{i}, \dot{\beta}_{i}$ track the desired signal as well, then $w_{i}=\left[\dot{\psi}_{i}-\dot{\beta}_{i}\right]$ is tracked. 
4.2 Fault Tolerant Controller Design

In order to eliminate the influence of the faults, a compensation controller is designed.

$\delta_{i a d}(t)=K_{i 2} \bar{m}_{i}(t)=\left[\begin{array}{ll}K_{i 2 \eta_{i}} & K_{i 2 m_{i}}\end{array}\right]\left[\begin{array}{c}\eta_{i}(t) \\ m_{i}(t)\end{array}\right]$

The whole control law $\delta_{i}(t)$ for the system is

$\delta_{i}(t)=\delta_{i 1}(t)+c_{i} \delta_{i a d}(t)$

where $c_{i}$ is a function that satisfies

$c_{i}= \begin{cases}0 & \text { if all actuators are fault-free } \\ 1 & \text { otherwise }\end{cases}$

Once the permanent fault occurs, the system stabilized by controller $\delta_{i}(t)$ becomes

$$
\begin{aligned}
& \left\{\begin{array}{l}
\dot{\bar{m}}_{i}(t)=\tilde{A} \bar{m}_{i}(t)+\tilde{B} \delta_{i a d}(t)+G y_{i r}(t) \\
n_{i}(t)=C \bar{m}_{i}(t)
\end{array}\right. \\
& \tilde{A}=\bar{A}+\bar{B} \rho_{i} K_{i 1}, \quad \tilde{B}=\bar{B} \rho_{i}
\end{aligned}
$$

The closed-loop system can be described as

$$
\left\{\begin{array}{l}
\dot{\bar{m}}_{i}(t)=\left(\tilde{A}+\tilde{B} K_{i 2}\right) \bar{m}_{i}(t)+G y_{i r}(t) \\
n_{i}(t)=C \bar{m}_{i}(t)
\end{array}\right.
$$

where $\widehat{A}=\tilde{A}+\tilde{B} K_{i 2}$.

Lemma 3 (Schur Complement) For a partitioned matrix $X=\left[\begin{array}{ll}X_{11} & X_{12} \\ X_{12}^{T} & X_{22}\end{array}\right]$, where $X_{11}$ is a block- matrix, then the following three conditions are equivalent:
a) $X<0$
b) $X_{11}<0, X_{22}-X_{12}^{T} X_{11}^{-1} X_{12}<0$
c) $X_{22}<0, X_{11}-X_{12} X_{22}^{-1} X_{12}^{T}<0$

Proof The proof is similar to [14], and thus is omitted.

Theorem 2 Given a constant $\gamma_{f}$, if there exists matrix $X=X^{T}>0, Y$, such that the following inequality holds

$$
\left[\begin{array}{ccc}
\tilde{A}^{T} X+X \tilde{A}+\tilde{B} Y+Y^{T} \tilde{B}^{T} & * & * \\
G & -I & * \\
C X & 0 & -\gamma_{f}^{2} I
\end{array}\right]<0
$$

Then compensate state feedback controller (19) exists, such that the error $\eta_{i}(t)$ is asymptotically stable. The gain of controller (19) is

$K_{i 2}=Y X^{-1}$

Proof According to [13], if

$\left[\begin{array}{ll}\widehat{A}^{T} P+P \widehat{A}+C^{T} C & P G \\ G^{T} P & -\gamma_{f}^{2} I\end{array}\right]<0$

holds, the system satisfies $\mathrm{H} \infty$ performance indicators, left-multiply and right-multiply matrix $\operatorname{diag}\left(P^{-1}, I\right)$,

$$
\left[\begin{array}{ll}
\hat{A}^{T} P+P \hat{A}+C^{T} C & P G \\
G^{T} P & -\gamma_{f}^{2} I
\end{array}\right]=\left[\begin{array}{ll}
P^{-1} \hat{A}^{T} P P^{-1}+P^{-1} P \hat{A} P^{-1}+P^{-1} C^{T} C P^{-1} & P^{-1} P G P^{-1} \\
P^{-1} G^{T} P P^{-1} & P^{-1}-\gamma_{f}^{2} I P^{-1}
\end{array}\right]<0
$$

Let $X=P^{-1}$, we have

$$
\begin{aligned}
& {\left[\begin{array}{ll}
X \hat{A}^{T}+\hat{A} X+X C^{T} C X & G X \\
X G^{T} & -\gamma_{f}^{2} I
\end{array}\right]<0} \\
& \Rightarrow\left[\begin{array}{ll}
X \hat{A}^{T}+\hat{A} X+\gamma_{f}^{2} X C^{T} C X & G^{T} \\
X & -I
\end{array}\right]<0
\end{aligned}
$$

while $\hat{A}=\tilde{A}+\tilde{B} K_{i 2}$,

$$
\begin{aligned}
& {\left[\begin{array}{ll}
X \hat{A}^{T}+\hat{A} X+\gamma_{f}^{2} X C^{T} C X & G^{T} \\
G & -I
\end{array}\right]} \\
& =\left[\begin{array}{ll}
X\left(\tilde{A}+\tilde{B} K_{i 2}\right)^{T}+\left(\tilde{A}+\tilde{B} K_{i 2}\right) X+\gamma_{f}^{2} X C^{T} C X & G^{T} \\
G & -I
\end{array}\right] \\
& <0
\end{aligned}
$$


then

$\left[\begin{array}{ll}X\left(\tilde{A}+\tilde{B} K_{i 2}\right)^{T}+\left(\tilde{A}+\tilde{B} K_{i 2}\right) X+\gamma_{f}^{2} X C^{T} C X & G^{T} \\ G & -I\end{array}\right]$
$=\left[\begin{array}{ll}X \tilde{A}^{T}+\tilde{A} X+X K_{i 2}^{T} \tilde{B}^{T}+\tilde{B} K_{i 2} X+\gamma_{f}^{2} X C^{T} C X & G^{T} \\ G & -I\end{array}\right]$
$<0$

Denote $Y=K_{i 2} X$

$\left[\begin{array}{cc}X \tilde{A}^{T}+\tilde{A} X+Y^{T} \tilde{B}^{T}+\tilde{B} Y+\gamma_{f}^{2} X C^{T} C X & G^{T} \\ G & -I\end{array}\right]<0$

By using Lemma 3,

$\left[\begin{array}{ccc}\tilde{A}^{T} X+X \tilde{A}+\tilde{B} Y+Y^{T} \tilde{B}^{T} & * & * \\ G & -I & * \\ C X & 0 & -\gamma_{f}^{2} I\end{array}\right]<0$

One can get

$$
\begin{aligned}
& {\left[\begin{array}{cc}
\tilde{A}^{T} X+X \tilde{A}+\tilde{B} Y+Y^{T} \tilde{B} & G^{T} \\
G & -I
\end{array}\right]} \\
& +\left[\begin{array}{c}
X^{T} C^{T} \\
0
\end{array}\right] \bullet \gamma_{f}^{2} I \bullet\left[\begin{array}{ll}
C X & 0
\end{array}\right]<0 \\
& \Rightarrow\left[\begin{array}{cc}
\tilde{A}^{T} X+X \tilde{A}+\tilde{B} Y+Y^{T} \tilde{B}+\gamma_{f}^{2} X^{T} C^{T} C X & G^{T} \\
G & -I
\end{array}\right]<0
\end{aligned}
$$

Then the proof is completed.

It can be seen that the proposed fault tolerant control method is decentralized since each UAV just needs to know the information of its neighbor [18], and the FTC scheme is needed only for the faulty UAV rather than the whole formation, this control strategy greatly reduces the amount of computation and the efficiency of achievement is extraordinary.

\section{FTC of Intermittent Faults}

The following lemma analyzes the UAV's behavior when there is an intermittent fault.

Lemma 4 If there exists an intermittent fault, even if asymmetric matrix $P_{i}^{\prime}$ and a positive define matrix $Q_{i}^{\prime}$ exist, the error $\eta_{i}$ of the inner-loop system may not be exponential stable.
Proof At the occurrence of intermittent faults, Eq. 15 changes into

$$
\begin{aligned}
\dot{V}_{i}(t)= & \dot{\eta}_{i}^{T}(t) P_{i}^{\prime} \eta_{i}(t)+\eta_{i}^{T}(t) P_{i}^{\prime} \eta_{i}(t) \\
= & 2 \eta_{i}^{T}(t) P_{i}^{\prime} G_{\eta_{i}} y_{i r}(t) \\
& +\eta_{i}^{T}(t)\left[A_{\eta_{i}}^{T} P_{i}^{\prime}+P_{i}^{\prime} A_{\eta_{i}}+\left(B_{\eta_{i}} K_{i 1_{\eta_{i}}} f_{i}\right)^{T} P_{i}^{\prime}\right. \\
& \left.+P_{i}^{\prime} B_{\eta_{i}} K_{i 1_{\eta_{i}}} f_{i}\right] \eta_{i}(t) \\
\leq 2\left|\eta_{i}^{T}(t)\right| P_{i}^{\prime} G_{\eta_{i}}\left|y_{i r}(t)\right| & \\
& +\eta_{i}^{T}(t)\left[A_{\eta_{i}}^{T} P_{i}^{\prime}+P_{i}^{\prime} A_{\eta_{i}}+\left(B_{\eta_{i}} K_{i 1_{\eta_{i}}} f_{i}\right)^{T} P_{i}^{\prime}\right. \\
& \left.+P_{i}^{\prime} B_{\eta_{i}} K_{i 1_{\eta_{i}}} f_{i}\right] \eta_{i}(t) \\
\leq & \eta_{i}^{T}(t)\left[A_{\eta_{i}}^{T} P_{i}^{\prime}+P_{i}^{\prime} A_{\eta_{i}}+\left(B_{\eta_{i}} K_{i 1_{\eta_{i}}} f_{i}\right)^{T} P_{i}^{\prime}\right. \\
\quad & \left.\quad P_{i}^{\prime} B_{\eta_{i}} K_{i 1_{\eta_{i}}} f_{i}\right] \eta_{i}(t)
\end{aligned}
$$

Then

$$
\begin{aligned}
Q_{i}^{\prime}= & A_{\eta_{i}}^{T} P_{i^{\prime}}+P_{i}^{\prime} A_{\eta_{i}}+\left(B_{\eta_{i}} K_{i 1_{\eta_{i}}} f_{i}\right)^{T} P_{i}^{\prime} \\
& +P_{i}^{\prime} B_{\eta_{i}} K_{i 1_{\eta_{i}}} f_{i}
\end{aligned}
$$

Equation 16 changes into

$\dot{V}_{i} \leq \lambda_{i 2}\left\|\eta_{i}(t)\right\|^{2}$

where $\lambda_{i 2}>0$ is the eigenvalue of $Q_{i}^{\prime}$. The proof is completed.

Let $P_{i j}(t)$ denote the probability for going from state $i(i=0,1)$ to state $j(j=0,1)$. The equations for these probabilities are

$$
\left\{\begin{aligned}
P_{0,1}(t) & =\frac{\lambda}{\lambda+\mu}\left(1-e^{-(\lambda+\mu) t}\right) \leq \frac{\lambda}{\lambda+\mu} \\
P_{0,0}(t) & =1-P_{0,1}(t)=\frac{\mu}{\lambda+\mu}+\frac{\lambda}{\lambda+\mu} e^{-(\lambda+\mu) t} \\
& \geq \frac{\mu}{\lambda+\mu}
\end{aligned}\right.
$$

Then during time period $[0, t)$, the time period the system keeps stable is $P_{0,0}(t) t$, and the time period the system becomes unstable is $P_{0,1}(t) t$. 
Define $P_{0,1} \triangleq \frac{\lambda}{\lambda+\mu}, P_{0,0} \triangleq \frac{\mu}{\lambda+\mu}$. It is clear that at each time instant, the probability of the healthy is more than $\underline{P}_{0,0}$, while the probability of faulty is less than $P_{0,1}[23,24]$. $E(\bullet)$ indicates the expectation.

Theorem 3 Consider system (10) with intermittent. There exists an initial condition of $\bar{m}_{i}(0)$ and $\delta_{i 1}$, such that the origin is asymptotically in probability, if

$\lambda_{i 2} \lambda<\lambda_{i 1} \mu$

Proof According to Eqs. 15 and 16,

$V_{i}(t) \leq e^{-\lambda_{i 1} \Delta t_{1}+\lambda_{i 2} \Delta t_{2}} V_{i}(0)$

while $-\lambda_{i 1} \Delta t_{1}+\lambda_{i 2} \Delta t_{2}$ can be described as $-\lambda_{i 1} P_{0,0}(t) t+\lambda_{i 2} P_{0,1}(t) t$

Then

$$
\begin{aligned}
E\left(V_{i}(t)\right) & \leq e^{\int_{0}^{t}\left(-\lambda_{i 1} P_{0,0}(\tau)+\lambda_{i 2} P_{0,1}(\tau)\right) d \tau} V_{i}(0) \\
& \leq e^{\left(-\lambda_{i 1} \frac{\mu}{\mu+\lambda}+\lambda_{i 2} \frac{\lambda}{\mu+\lambda}\right) t} V_{i}(0) \quad \forall t \geq 0
\end{aligned}
$$

If $-\lambda_{i 1} \frac{\lambda}{\lambda+\mu}+\lambda_{i 2} \frac{\mu}{\lambda+\mu}<0$, one can have $\lambda_{i 2} \lambda<$ $\lambda_{i 1} \mu$. Condition (27) ensures that $E\left(V_{i}(t)\right)<$ $V_{i}(0)$, which means that the control law (12) is always available in probability. Hence, we can have $\lim _{t \rightarrow \infty} E\left(V_{i}(t)\right)=0$. The proof is completed.

Condition (27) provides an explicit relation among healthy and faulty situations for the maintenance of the stability, which implies that the healthy situation can compensate for the negative effect of faulty situations provided that $\lambda_{i 1}$ and $\mu$ are large enough compared with $\lambda_{i 2}$ and $\lambda$. Note that any active FTC design is not needed. Such a result can be combined with other FTC design method to improve the reliability of the flight control system with respect to intermittent faults, and to make the FTC scheme more flexible.

\section{Simulation Results}

In the simulation, the formation is composed of 5 UAVs as is shown in Fig. 3. Details of such formation model can be found in [1].

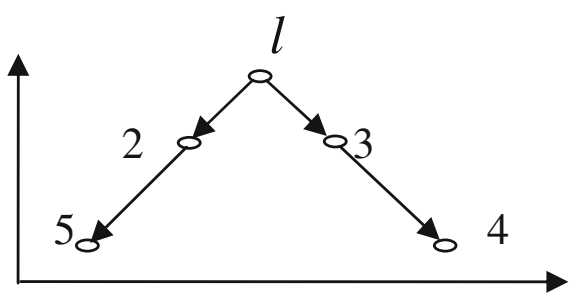

Fig. 3 Topology of 5 UAVs in formation

The desired angle and velocity are $V^{*}=30 \mathrm{~m} / \mathrm{s}$, $w^{*}=0.5 \mathrm{rad} / \mathrm{s}$. As for the inner-loop variables, $\psi^{*}=32 \mathrm{deg} / \mathrm{s}, \beta^{*}=6 \mathrm{deg} / \mathrm{s} \mathrm{UAV} 2$ is faulty.

The system matrices take the form:

$$
\begin{aligned}
A & =\left[\begin{array}{ll}
A_{1} & 0 \\
0 & A_{2}
\end{array}\right], B=\left[\begin{array}{ccc}
B_{1} & 0 \\
0 & B_{2}
\end{array}\right] \\
A_{1} & =\left[\begin{array}{cccc}
-0.0334 & -2.977 & 0.00 & -9.81 \\
-0.0016 & -4.133 & 0.98 & 0 \\
0.0077 & -140.2 & -4.435 & 0 \\
0 & 0 & 1 & 0
\end{array}\right] \\
A_{2} & =\left[\begin{array}{cccc}
-0.732 & 0.0143 & -0.996 & 0.0706 \\
-893 & -9.059 & 2.044 & 0 \\
101.673 & 0.0186 & -1.283 & 0 \\
0 & 0 & 1 & 0
\end{array}\right]
\end{aligned}
$$$$
B_{1}=\left[\begin{array}{cc}
-1.075 & 0.2453 \\
0.3470 & -4.133 \\
-140.22 & 0 \\
0 & 0
\end{array}\right] \text {, }
$$$$
B_{2}=\left[\begin{array}{cc}
0 & 0.244 \\
328.653 & 308.498 \\
47.5280 & 102.891 \\
0 & 0
\end{array}\right]
$$$$
C=\left[\begin{array}{cccccccc}
1 & 0 & 0 & 0 & 0 & 0 & 0 & 0 \\
0 & 0 & 0 & 0 & -1 & 0 & 0 & 1
\end{array}\right]
$$$$
\bar{A}=\left[\begin{array}{cc}
0 & -S C \\
0 & A
\end{array}\right], \bar{B}=\left[\begin{array}{l}
0 \\
B
\end{array}\right],
$$ 


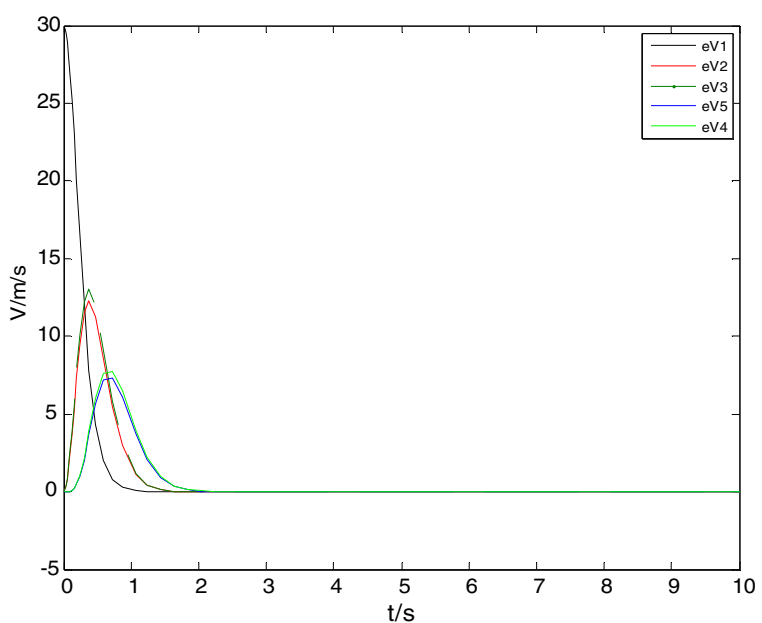

Fig. 4 Velocity error of normal system

\section{Choose}

$S=\left[\begin{array}{ll}1 & 0 \\ 0 & 1\end{array}\right]$

By using LMI, one obtain state feedback control gain of normal system $K_{21}=\left[\begin{array}{cc}K_{21_{1}} & 0 \\ 0 & K_{21_{2}}\end{array}\right]$

$$
\begin{gathered}
K_{21_{1}}=\left[\begin{array}{ccccc}
-1.1350 & 0.1979 & -1.0130 & 0.1079 & 0.5988 \\
86.7668 & -25.9686 & 4.0673 & 1.1980 & 26.8821
\end{array}\right] \\
K_{21_{2}}=\left[\begin{array}{ccccc}
13.5138 & 2.8178 & -0.0577 & 0.4109 & -0.5078 \\
-14.3884 & -0.1090 & 0.0292 & -0.4442 & 0.2494
\end{array}\right]
\end{gathered}
$$

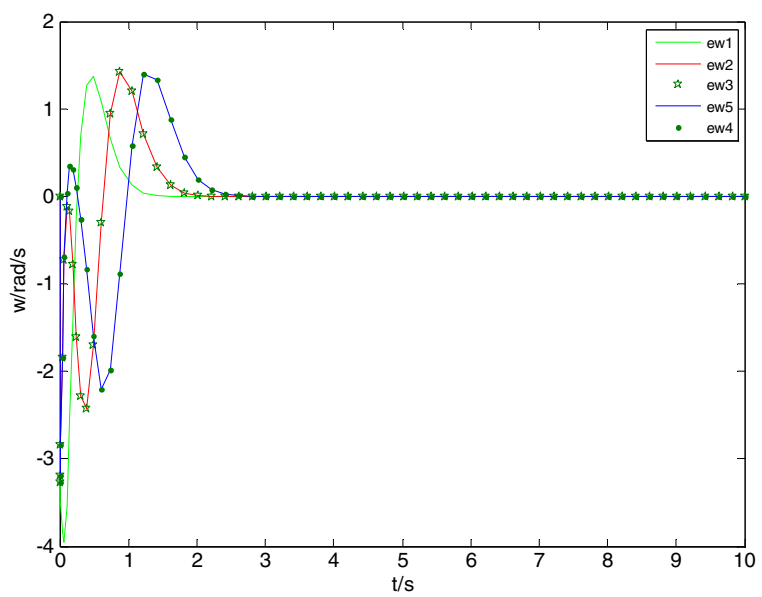

Fig. 5 Angular velocity error of normal system

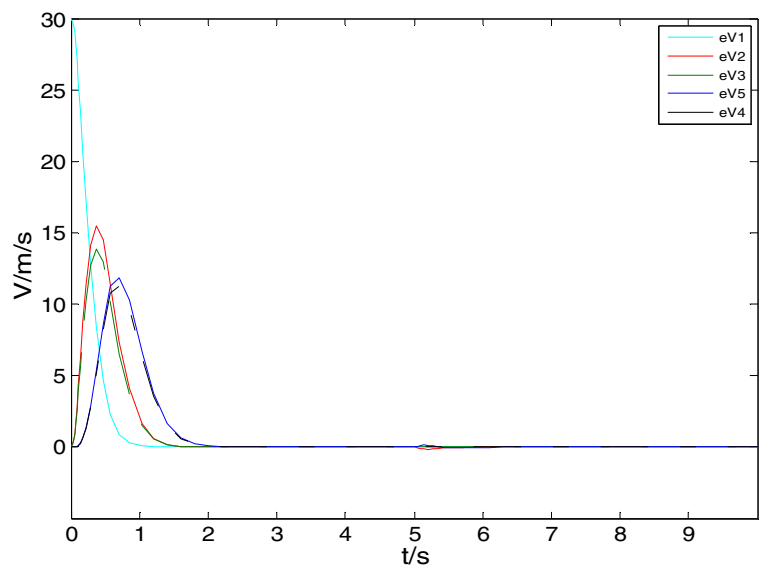

Fig. 6 Velocity error under incipient fault

As for the normal system, the velocity error and angular velocity error are showed in Figs. 4 and 5.

\subsection{Permanent Faults}

The incipient permanent fault happens at $5 \mathrm{~s}$.

$\rho_{2}=\operatorname{diag}[0.95,0.99,0.95,0.97]$

Compensate control gain is $K_{22}=\left[\begin{array}{cc}K_{22_{1}} & 0 \\ 0 & K_{22_{2}}\end{array}\right]$

$$
\begin{gathered}
K_{22_{1}}=\left[\begin{array}{ccccc}
-0.0598 & 0.0104 & -0.0534 & 0.0057 & 0.0315 \\
0.8764 & -0.2623 & 0.0411 & 0.0121 & 0.2716
\end{array}\right] \\
K_{22_{2}}=\left[\begin{array}{ccccc}
0.7112 & 0.1483 & -0.0030 & 0.0216 & -0.0267 \\
-0.1453 & -0.0011 & 0.0003 & -0.0045 & 0.0025
\end{array}\right]
\end{gathered}
$$

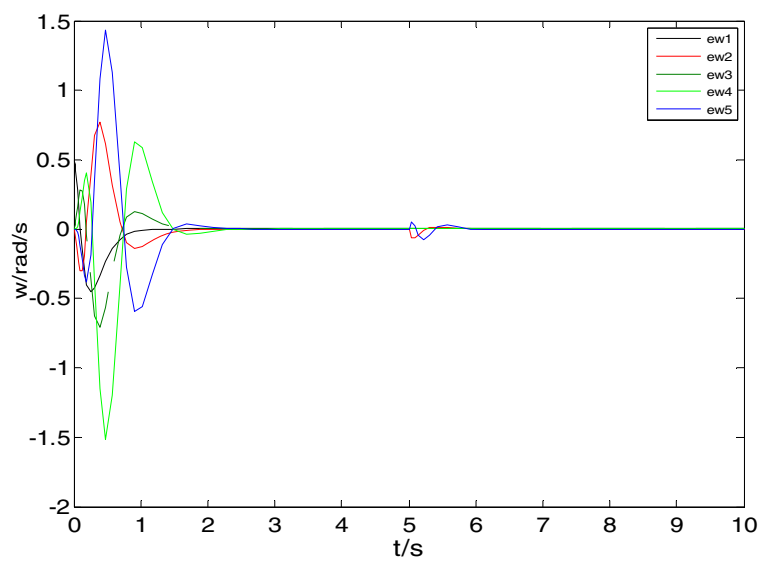

Fig. 7 Angular velocity error under incipient fault 


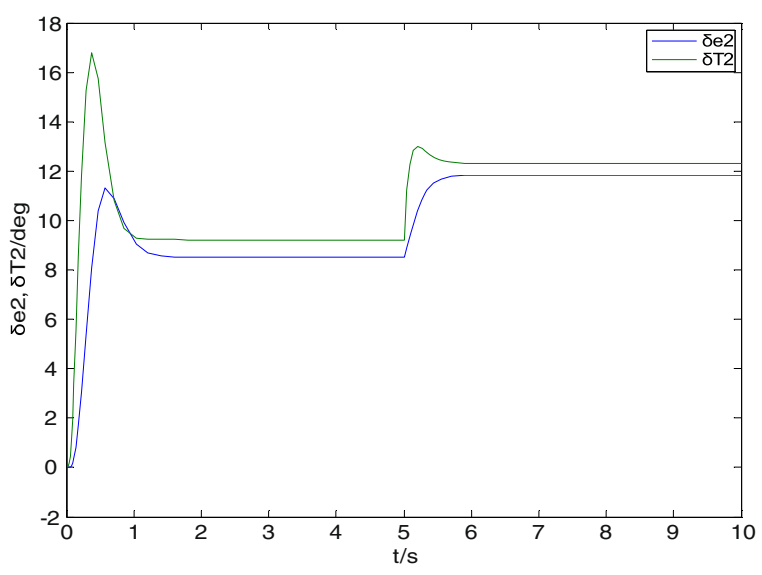

Fig. 8 Change of control surfaces $\delta_{e 2}, \delta_{T 2}$ under incipient fault

Figures 6 and 7 show velocity and angular velocity error under incipient fault respectively. Figures 8 and 9 show the changes of control surfaces $\delta_{2 e}, \delta_{2 T}$ and $\delta_{2 a}, \delta_{2 r}$ under incipient fault respectively.

The severe permanent fault happens at $5 \mathrm{~s}$.

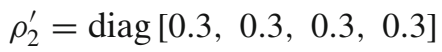

Compensate control gain is $K_{22}=\left[\begin{array}{cc}K_{22_{1}} & 0 \\ 0 & K_{22_{2}}\end{array}\right]$ $K_{22_{1}}=\left[\begin{array}{ccccc}-2.6484 & 0.4598 & -2.3638 & 0.2517 & 1.3971 \\ 202.4558 & -60.5935 & 9.4904 & 2.7953 & 62.7249\end{array}\right]$

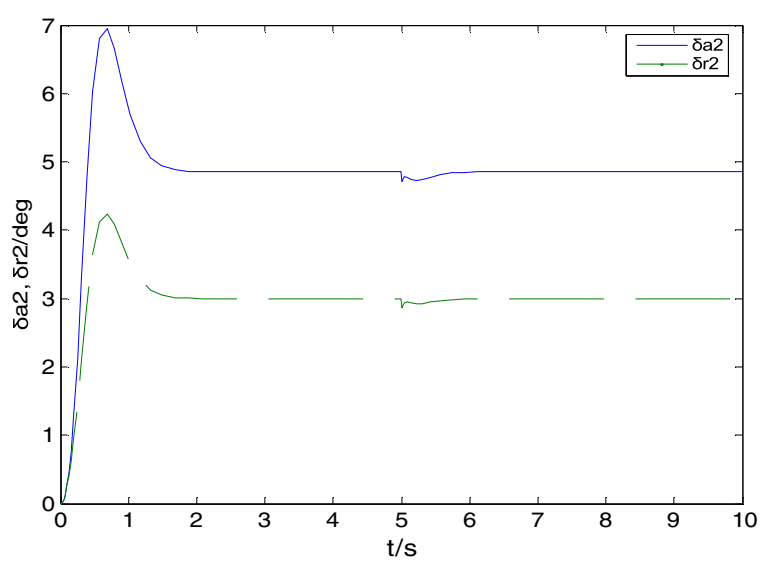

Fig. 9 Change of control surfaces $\delta_{e 2}, \delta_{r 2}$ under incipient fault

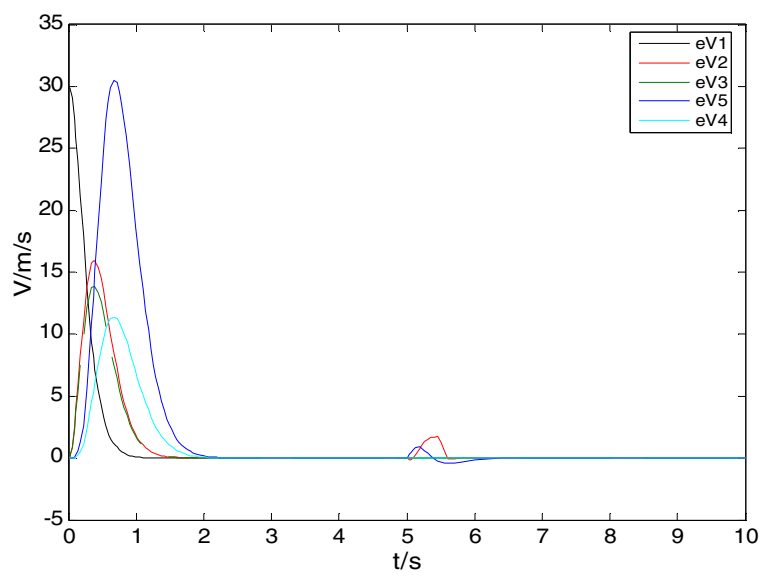

Fig. 10 Velocity error under severe fault

$K_{22_{2}}=\left[\begin{array}{ccccc}31.5320 & 6.5748 & -0.1345 & 0.9587 & -1.1848 \\ -33.5729 & -0.2542 & 0.0682 & -1.0366 & 0.5818\end{array}\right]$

Figures 10 and 11 show velocity and angular velocity error of severe fault respectively. Figures 12 and 13 show changes of control surfaces $\delta_{2 e}, \delta_{2 T}$ and $\delta_{2 a}, \delta_{2 r}$ under severe fault respectively.

\subsection{Intermittent Faults}

At the occurrence of intermittent fault, the fault's appearance and disappearance are showed in

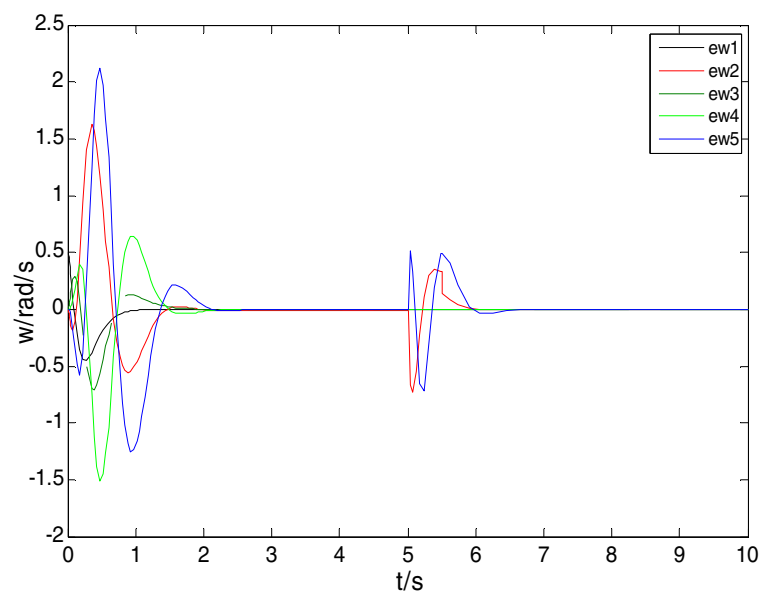

Fig. 11 Angular velocity error under severe fault 


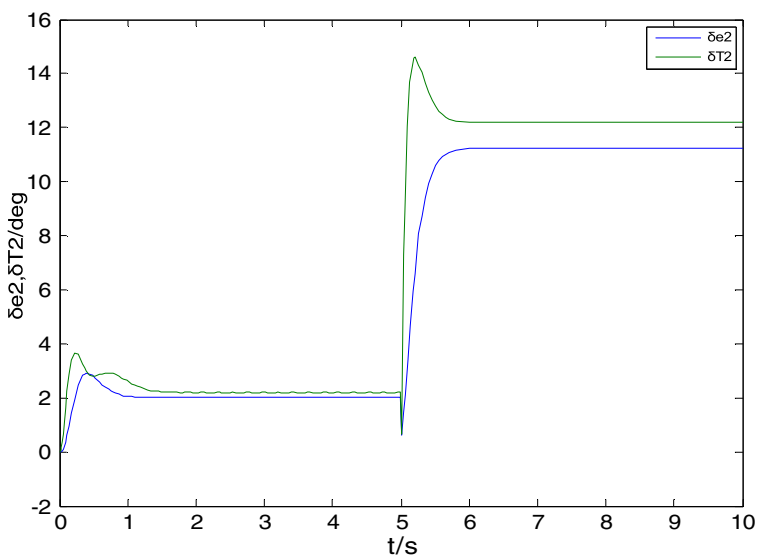

Fig. 12 Change of control surfaces $\delta_{e 2}, \delta_{T 2}$ under severe fault

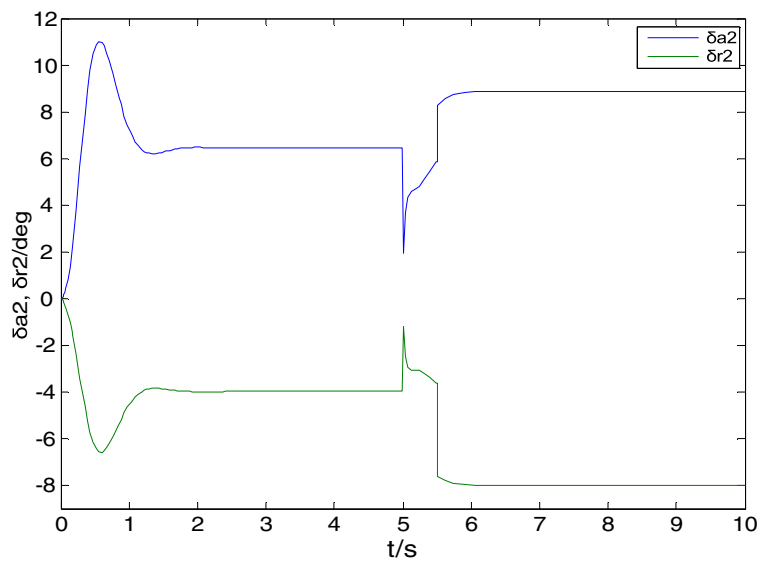

Fig. 13 Change of control surfaces $\delta_{a 2}, \delta_{r 2}$ under severe fault

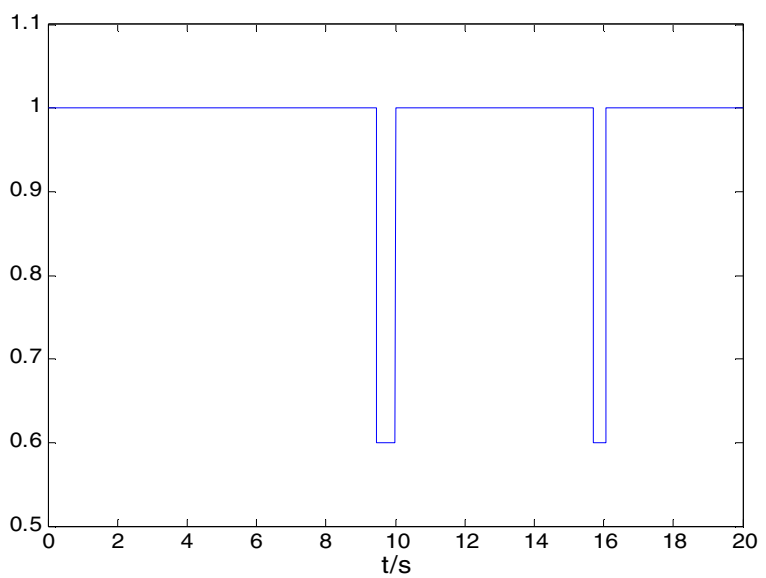

Fig. 14 Intermittent fault model

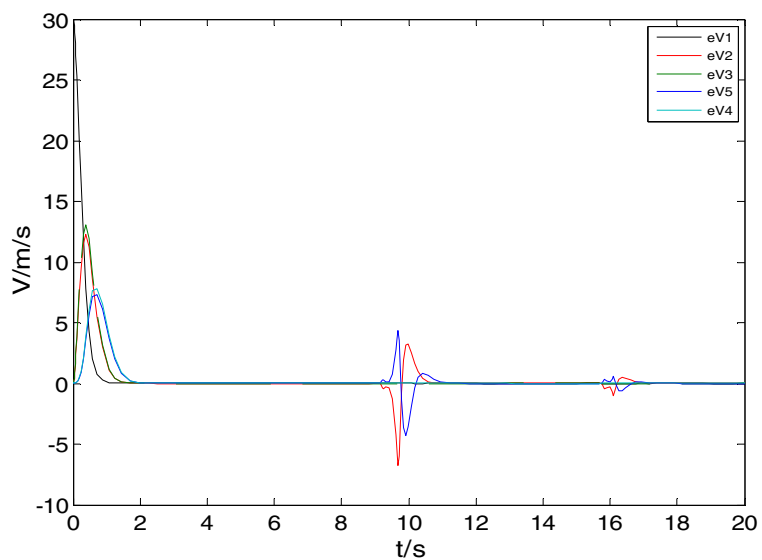

Fig. 15 Velocity error of intermittent fault

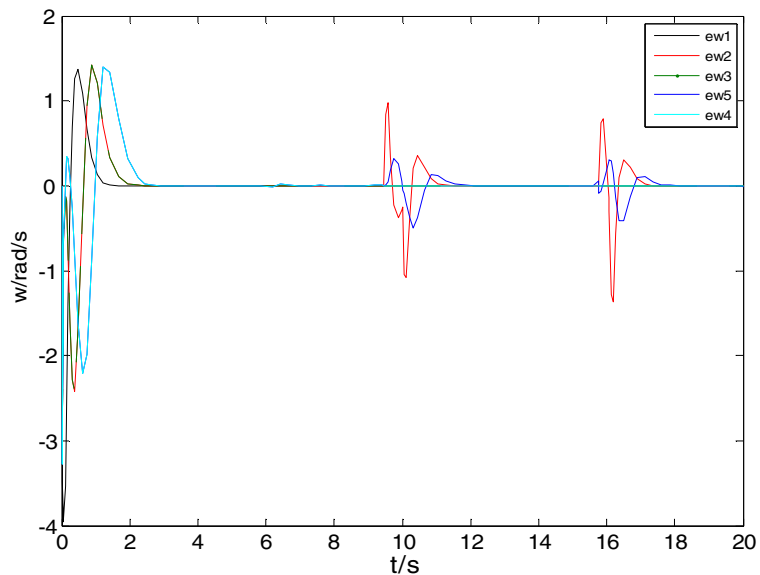

Fig. 16 Angular velocity error of intermittent fault

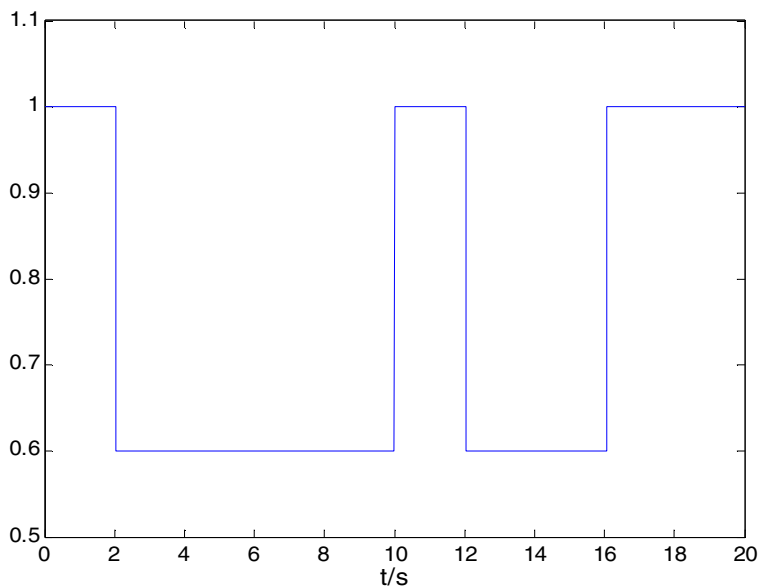

Fig. 17 Changing fault model 


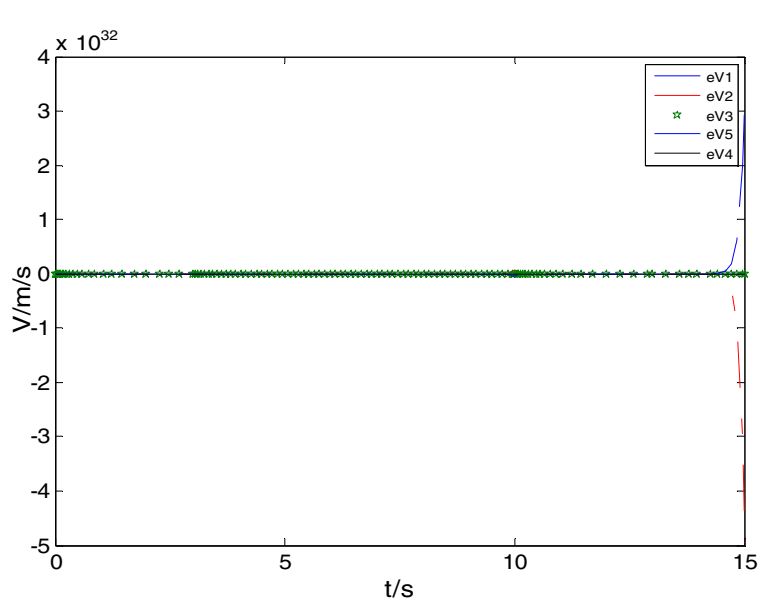

Fig. 18 Velocity error of intermittent fault

Fig. 14. The faults happened at $9 \mathrm{~s}$ and $15.5 \mathrm{~s}$ respectively and disappeared at $10 \mathrm{~s}$ and $16 \mathrm{~s}$ respectively.

According to Figs. 14, 15 and 16, one can see that the intermittent model satisfies the probability proposed by Theorem 1 . And the faulty system is stable without designing a new controller.

By changing the fault model, one can see from Figs. 17, 18 and 19 that the fault model does not satisfy the probability, so the system becomes unstable. The first time the fault happens at $2 \mathrm{~s}$ and disappears at $10 \mathrm{~s}$, the second time the fault happens at $12 \mathrm{~s}$ and disappears at $16 \mathrm{~s}$.

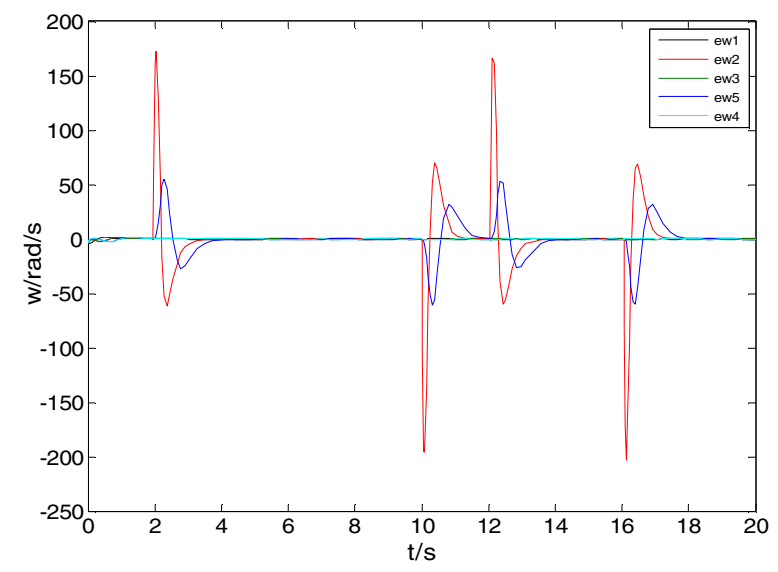

Fig. 19 Angular velocity error of intermittent fault

\section{Conclusion}

This paper considers the FTC problem of UAVs formation in the presence of permanent and intermittent faults. FTC is achieved in each individual $\mathrm{UAV}$, the future work still focus on cooperative FTC design under which the FTC goal can be achieved by cooperation among UAVs.

Open Access This article is distributed under the terms of the Creative Commons Attribution License which permits any use, distribution, and reproduction in any medium, provided the original author(s) and the source are credited.

\section{References}

1. Reyna, V.P.: Automation of Formation Flight Control. Air Force Inst of Tech Wright-Patterson AFB OH School of Engineering (1994)

2. Giulietti, F., Pollini, L, Innocenti, M.: Autonomous formation flight. IEEE Contr. Syst. Mag. 20(6), 34-44 (2000)

3. Pachter, M., Huang, Y.S.: Fault tolerant flight control. J. Guid. Control Dyn. 26(1), 151-160 (2003)

4. Song, Y.D.: Fault-tolerant and reconfigurable control of unmanned aerial vehicles (UAVS). North Carolina Agricultural and Technial State University, Dept. of Electrical Engineering, Greensboro (2008)

5. Ye, D., Yang, G.H.: Adaptive fault-tolerant tracking control against actuator faults. In: Proceedings of the 2006 American Control Conference Minneapolis, Minnesota, USA (2006)

6. Ye, D., Yang, G.H.: Adaptive fault-tolerant tracking control against actuator faults with application to flight control. IEEE Trans. Control Syst. Technol. 14(6), 1088-1096 (2006)

7. Zhang, X.N., Wang, J.L., Yang, G.H.: Adaptive faulttolerant tracking controller design against actuator stuck faults. In: 2012 24th Chinese Control and Decision Conference (CCDC) (2012)

8. Ismaeel, A., Bhatnagar, R.: Test for detection \& location of intermittent faults in combinational circuits. IEEE Trans. Reliab. 46(2), 269-274 (1997)

9. Hsu, Y.T., Hsu, C.F.: Novel model of intermittent faults for reliability and safety measures in long-life computer systems. Int. J. Electron. 71(6), 917-937 (1991)

10. Su, S., Koren, Y.H., Malaiya, Y.K.: A continuousparameter Markov model and detection procedures for intermittent faults. IEEE Trans. Comput. C-27(6), 567-570 (1978) 
11. Breuer, M.A.: Testing for intermittent faults in digital circuits. IEEE Trans. Comput. C-22, 241-246 (1973)

12. Kamal, S., Page, C.V.: Intermittent faults: a model and a detection procedure. IEEE Trans. Comput. C-23, 713-719 (1974)

13. Chen, W., Chowdhury, N.: Design of sliding mode observers with sensitivity to incipient faults. In: 16th IEEE International Conference on Control Applications Part of IEEE Multi-Conference on Systems and Control Singapore (2007)

14. Xie, L.H.: Output feedback control of systems with parameter uncertainty. Int. J. Control 63(4), 741-750 (1996)

15. Parzen, E.: Stochastic Processes. Holden, New York (1962)

16. Skorohod, A.V.: Asymptotic methods in the theory of stochastic differential equations. American Mathematical Society (2009)

17. Yang, H., Jiang, B., Zhang, Y.: Tolerance of intermittent faults in spacecraft attitude control: switched system approach. IET Control Theory Appl. 6(13), 2049-2056 (2012)

18. Tamas, K., Balint, V., Francesco, B.: Hybrid decentralized receding horizon control of vehicle formations.
In: Proceedings of the 2006 American Control Conference Minneapolis, Minnesota, USA (2006)

19. Sun, F.L., Guan, Z.H., Zhang, X.H., Chen, J.C.: Exponential-weighted input-to-state stability of hybrid impulsive switched systems. IET Control Theory Appl. 6(3), 430-436 (2012)

20. Lars, G.: Input-to-state dynamical stability and its Lyapunov function characterization. IEEE Trans. Autom. Control 47(9), 1499-1504 (2002)

21. Nima, Y., Pierdomenico, P., Michel, D.: Input-to-state stability of time-delay systems: a link with exponential stability. IEEE Trans. Autom. Control 53, 6 (2008)

22. Zhou, Z., Yang, C., Zhang, Q., Cai, M.: Brief paper Input-to-state stability for descriptor systems with nonlinear perturbations. IET Control Theory Appl. 5(13), 1561-1567 (2010)

23. Xuerong, M.: Stability of stochastic differential equations with Markovian switching. Stoch. Process. Appl. 79(1), 45-67 (1999)

24. Yang, H., Jiang, B., Cocquempot, V.: Fault Tolerant Control Design for Hybrid Systems. Lecture Notes in Control and Information Sciences, vol. 397 (Paperback)

25. Pachpatte, B.G.: A note on Gronwall-Bellman inequality. J. Math. Anal. Appl. 44(3), 758-762 (1973) 